\title{
Definición de medicamento genérico ¿un fin o un medio? Análisis de la regulación en 14 países de la Región de las Américas
}

\author{
Claudia Patricia Vacca González, ${ }^{1}$ James F. Fitzgerald ${ }^{2}$ y Jorge A. Z. Bermúdez ${ }^{3}$
}

Forma de citar

Vacca González CP, Fitzgerald JF, Bermúdez JAZ. Definición de medicamento genérico ¿un fin o un medio? Análisis de la regulación en 14 países de la Región de las Américas. Rev Panam Salud Publica. 2006;20(5):314-23.

RESUMEN Objetivos. Caracterizar la situación y las tendencias regulatorias relacionadas con los medicamentos competidores en 14 países de América Latina y el Caribe.

Métodos. Entre julio de 2004 y abril de 2005 se colectó la información sobre las regulaciones y politicas nacionales que establecían o contenían la definición de medicamento genérico y de los términos asociados en 14 países de América Latina y el Caribe (Argentina, Barbados, Bolivia, Brasil, Colombia, Costa Rica, Ecuador, Guatemala, México, Nicaragua, Panamá, Paraguay, Perú y Venezuela). Además, se recabó información sobre los incentivos para registrar y producir medicamentos competidores, la promoción del uso de la denominación común internacional (DCI), la regulación de los estudios de bioequivalencia y la sustitución de los medicamentos prescritos por alternativas de menor precio al público.

Resultados. Se observaron tres tendencias regulatorias: los países que favorecen la financiación de medicamentos competidores, la promoción extendida del uso de la DCI y no ponen restricciones a la sustitución de medicamentos innovadores por competidores (Argentina, Colombia, Costa Rica, Ecuador y Paraguay); los que cuentan con un esquema orientado a la demostración de la equivalencia terapéutica, es decir, los países restringen la sustitución de los medicamentos originales mediante una lista de medicamentos competidores autorizados que deben prescribirse bajo su DCI y llevar rótulos distintivos (Brasil, México, Panamá y Venezuela); y los que se encuentran en una etapa incipiente de su proceso de regulación (Barbados, Bolivia, Guatemala, Nicaragua y Perú). Esta variedad ocasiona dificultades en la caracterización de los mercados farmacéuticos y puede generar segmentaciones ficticias.

Conclusiones. Los esfuerzos de armonización deberán tomar en cuenta la posible relación entre las definiciones adoptadas por los países, el desarrollo de los mercados farmacéuticos nacionales y sus políticas de estímulo a la competencia.

Palabras clave Denominaciones comunes internacionales para las sustancias farmacéuticas, medicamentos genéricos, control de medicamentos y narcóticos, Américas.
Instituto Nacional de Vigilancia de Medicamentos y Alimentos, Universidad Nacional de Colombia, Santa Fe de Bogotá, Colombia. La correspondencia se debe dirigir a Claudia P. Vacca, Calle 4 No. $37-$ 85 Apto. 106, Plazuelas de Tibaná II, Santa Fe de Bogotá, Colombia. Correos electrónicos: cpvacag@ unal.edu.co, cvacag@invima.gov.co
2 Unidad de Medicamentos Esenciales, Vacunas y Tecnologías en Salud, Organización Panamericana de la Salud, Brasilia, Brasil.

3 Unidad de Medicamentos Esenciales, Vacunas y Tecnologías en Salud, Organización Panamericana de la Salud, Washington, D.C., Estados Unidos de América.
Las recomendaciones internacionales en políticas farmacéuticas establecen la necesidad de promover la competencia en la oferta de medicamentos como una importante estrategia para 
mejorar el acceso a los mismos. Esto tiene un efecto benéfico sobre los precios y sobre el desarrollo de capacidades nacionales de producción, en especial de medicamentos esenciales (1-3).

La consolidación de la competencia en el mercado farmacéutico se hace posible al caducar los derechos exclusivos asociados con los mecanismos del sistema de propiedad intelectual. Aunque las normas de propiedad intelectual en el campo farmacéutico se aplican en los Estados Unidos y algunos países europeos desde hace más de 50 años (4), su aplicación en América Latina, el Caribe y la mayoría de los países en desarrollo comenzó en 1995, después de la creación de la Organización Mundial del Comercio (OMC) y la adopción del Acuerdo sobre los Aspectos de los Derechos de Propiedad Intelectual Relacionado con el Comercio (ADPIC) (5).

En América Latina, los mercados de medicamentos competidores se consolidaron antes de la adopción de los acuerdos y normas de propiedad intelectual vigentes $(4,5)$. Por lo tanto, resultó difícil adoptar la definición clásica de que medicamento genérico es el que se comercializa después de haber caducado las patentes que lo amparaban (6). En el presente trabajo se utilizará el término medicamento competidor para referirse a los llamados medicamentos genéricos.

Las políticas de promoción de la competencia en el sector farmacéutico suelen ofrecer ventajas tributarias e incentivos para el registro, la producción y la promoción del uso de la denominación común internacional (DCI), especialmente para la prescripción, la información comparativa de los precios y las campañas balanceadas de información dirigidas a los médicos, farmacéuticos o consumidores. De esta forma se persigue mejorar no solo la aceptación, sino también la regulación estricta de los requisitos de calidad cuando se promueve la sustitución del medicamento prescrito por una alternativa de menor precio al público.

Aunque la calidad es un componente general de las políticas farmacéuticas, es frecuente que se asocie más con las que incentivan el uso de los medica- mentos competidores, ya que en muchos países está generalizado el criterio de que los medicamentos competidores son de peor calidad que los llamados originales, es decir, los producidos por el laboratorio que realizó la investigación y desarrolló el principio activo. Conviene aclarar que la calidad farmacéutica se garantiza mediante los sistemas de aseguramiento de la calidad, cuya columna vertebral son las buenas prácticas de fabricación. Estos dos elementos son requisitos obligatorios para la producción de medicamentos, tanto de los competidores como de los originales (7).

Lamentablemente, se tiende a pensar de forma errónea que las pruebas de bioequivalencia (BE) son el único estándar de calidad de los medicamentos competidores y aunque la exigencia de realizar pruebas de BE forma parte de las políticas de competencia en el sector farmacéutico su reglamentación difiere de un país a otro (8). En la Región de las Américas se observa una tendencia a exigir la realización de las pruebas de $\mathrm{BE}$ en dependencia del riesgo sanitario específico de cada producto (9).

Si bien cada país ha establecido incentivos para la producción, la comercialización y el uso de los medicamentos competidores, se observan grandes variaciones entre ellos, particularmente en lo relacionado con la definición de medicamento genérico $(10,11)$ y en cuanto a la exigencia de realizar pruebas de BE $(10,12)$.

El objetivo del presente trabajo es caracterizar la situación y las tendencias regulatorias relacionadas con los medicamentos competidores en 14 países de América Latina y el Caribe.

\section{MATERIALES Y MÉTODOS}

Se seleccionó una muestra de conveniencia de 14 países de América Latina y el Caribe (Argentina, Barbados, Bolivia, Brasil, Colombia, Costa Rica, Ecuador, Guatemala, México, Nicaragua, Panamá, Paraguay, Perú y Venezuela) considerando la dinámica de esos países en el desarrollo regulatorio de medicamentos, la presencia de con- sultores de la Organización Panamericana de la Salud (OPS) y el grado de desarrollo de proyectos, programas y políticas relacionados con los medicamentos. La imposibilidad de acceder a la información regulatoria de Haití, previsto inicialmente en la muestra, obligó a excluirlo del estudio.

Entre julio de 2004 y abril de 2005 se colectó la información sobre las regulaciones y políticas nacionales que establecían o contenían la definición de medicamento genérico y de los términos asociados. Además, se recabó información sobre los incentivos establecidos en cada país para registrar y producir medicamentos competidores, promover el uso de la DCI en las diferentes etapas de su comercialización, regular los estudios de $\mathrm{BE}$ y sustituir los medicamentos prescritos por alternativas de menor precio al público. En todos los casos se intentó identificar las diferencias, incentivos y restricciones asociadas con el uso de la DCI en los documentos regulatorios y oficiales.

La información fue recolectada por los profesionales técnicos de la OPS en los países estudiados, con el apoyo de las autoridades nacionales y de expertos en el tema, mediante un modelo de recolección de información diseñada en MS Excel (Microsoft Corporation, Redmond, Estados Unidos de América). Se registró cada aspecto analizado según el tipo de documento regulatorio (decreto, ley, resolución, política o documentos oficiales y referencias detalladas).

Con la excepción de Ecuador, la información obtenida sobre la regulación de los medicamentos competidores fue fragmentada y de difícil consulta. Las variables evaluadas se presentan en el cuadro 1.

\section{RESULTADOS}

Se colectó la información completa de 11 países (Argentina, Barbados, Brasil, Colombia, Costa Rica, Ecuador, Guatemala, México, Panamá, Paraguay y Perú) e información parcial de otros tres (Bolivia, Nicaragua y Venezuela) (cuadro 1). Se logró recolectar 77\% del total de los parámetros previstos. La in- 
CUADRO 1. Comparación de los criterios regulados en los países estudiados. Abril, 2005

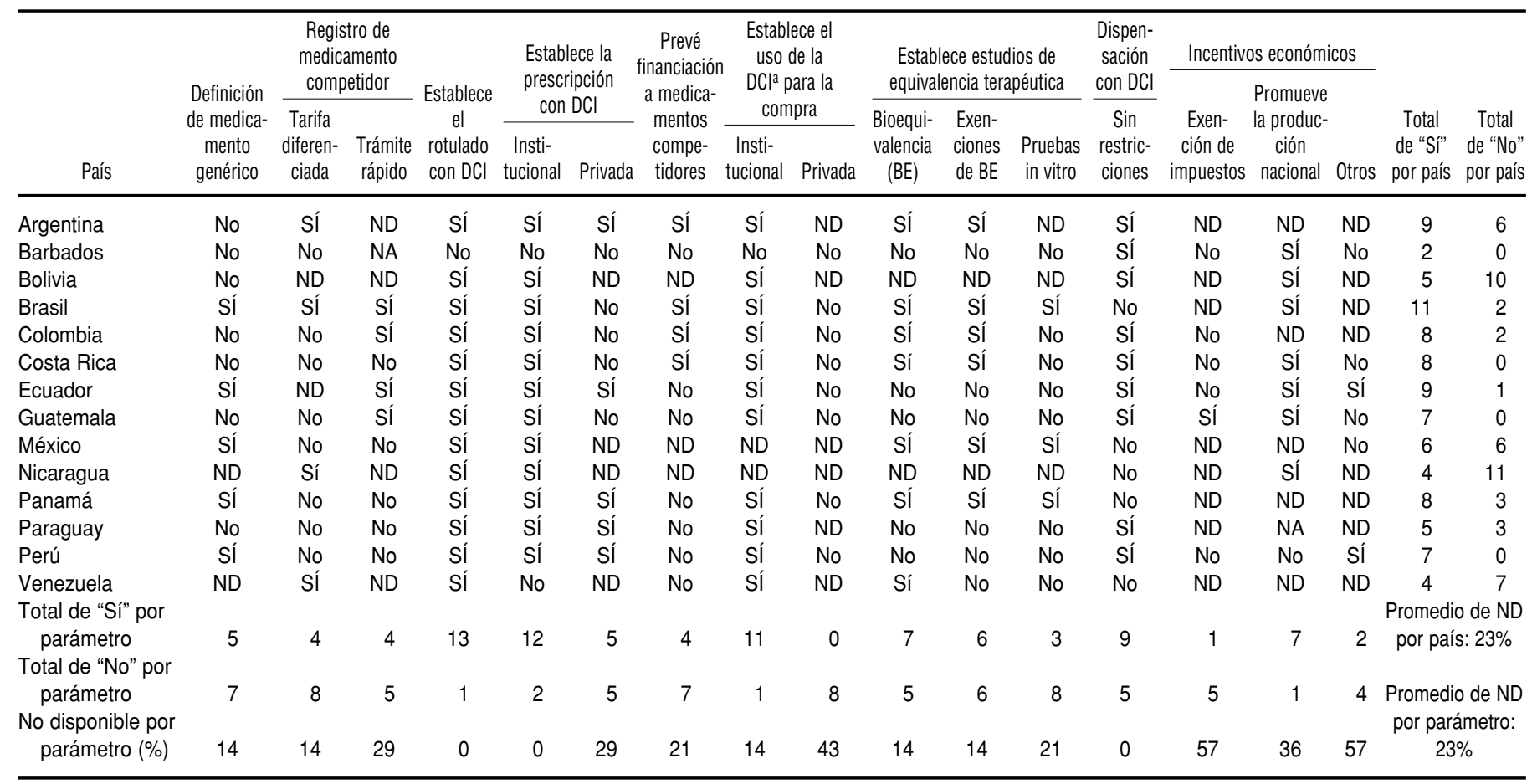

a DCl: denominación común internacional; Sí: existe regulación; No: no existe regulación; NA: no es aplicable; ND: no disponible.

formación que con mayor frecuencia no estuvo disponible fue la relacionada con los incentivos económicos.

\section{Exigencia y promoción del uso de la DCI}

Los aspectos que con mayor frecuencia estuvieron recogidos en las regulaciones fueron la obligatoriedad de utilizar la DCI en el rotulado de los medicamentos, lo cual se constató en 13 de los 14 países analizados (13/14), seguido de la obligatoriedad de hacer la prescripción empleando la DCI en el ámbito institucional público (12/14) y en los procesos de adquisición institucional de medicamentos (11/14) (cuadro 1).

\section{Incentivos al registro}

No hubo información disponible sobre los incentivos económicos que se ofrecen por registrar medicamentos competidores en cinco países (Argentina, Bolivia, Ecuador, Nicaragua y Venezuela). En general, los datos disponibles indican que este aspecto no está reflejado suficientemente en los documentos regulatorios nacionales (cuadro 1).

\section{Financiación que privilegia los medicamentos competidores}

En Argentina (13-15), Colombia (16) y Brasil (17), la financiación de medicamentos otorga ventajas a los competidores. En el sistema de salud de Costa Rica, que tiene una cobertura universal, se garantiza la disponibilidad de los medicamentos competidores (18). Aunque en Ecuador el sistema de salud no es de cobertura universal, llama la atención que en su sistema público de salud sea obligatoria la provisión de medicamentos competidores (19).

\section{Exigencia de pruebas de bioequivalencia}

Siete de los 15 países analizados, han regulado la exigencia de realizar estu- dios de BE. De ellos, en las regulaciones de Brasil (20), México (21) y Panamá (22) se establece la posibilidad de presentar pruebas in vitro como alternativa a la realización de estudios in vivo.

\section{Promoción de la sustitución}

En la mayoría de los países (9/14) no existen restricciones a la sustitución del medicamento prescrito por un medicamento competidor de menor precio. En cinco de los países analizados (Brasil, México, Nicaragua, Panamá y Venezuela), las restricciones a la sustitución se presentan mediante listas oficiales.

\section{Definición de medicamento genérico y de términos asociados}

Se observó una gran variedad en las definiciones. En los documentos regulatorios de algunos países no se define qué es un medicamento genérico y se adoptan otros términos (Argentina, Colombia y Paraguay), mientras en otros simplemente se consideran com- 
petidores los medicamentos que se comercializan con la DCI (Bolivia, Costa Rica, Panamá y Perú). En el resto de los países, los documentos regulatorios no contienen definición alguna (cuadro 2).

En Colombia se utiliza el término "medicamento competidor", mientras en Argentina se adoptó el término "medicamento similar". Llama la atención que aunque en Costa Rica se adoptó el término "medicamento o producto farmacéutico multiorigen", utilizado por la Organización Mundial de la Salud (OMS) $(11,23)$, la definición que ofrece no coincide exactamente con la sugerida por la OMS (cuadro 2).

Las definiciones de medicamento genérico empleadas en Brasil y México presentan leves diferencias e incorporan la obligatoriedad de demostrar la equivalencia terapéutica, los derechos de patente vencidos y el uso de la DCI en su comercialización (cuadro 2).

Podría decirse que las definiciones de medicamento genérico estudiadas varían según su forma de comercialización (si se comercializa con una marca, la DCI u otra denominación común aprobada), la exigencia o no de realizar pruebas de equivalencia terapéutica (ya sean de BE u otras) y el momento de comercialización (si se realiza después de vencidos los derechos de patente $u$ otras formas de exclusividad). En el cuadro 3 se resumen los criterios utilizados por los diferentes países en sus definiciones de medicamento genérico. Algunos países, como Perú, solo incorporan la categoría de comercialización con DCI en su definición de medicamento genérico (cuadro 2).

\section{DISCUSIÓN}

\section{Promoción del uso de la DCI}

El uso de la DCI en el proceso de comercialización de medicamentos es el mecanismo más ampliamente recomendado para promover la competencia, corregir las imperfecciones del mercado farmacéutico, propiciar el uso adecuado de los medicamentos y reducir la confusión derivada de la proliferación de marcas registradas de medicamentos (3).

Resulta interesante el desarrollo de este mecanismo en los documentos regulatorios de los países estudiados. Sólo Barbados no exige la DCI en el rotulado de los medicamentos como estrategia para incentivar la competencia en el mercado farmacéutico. La prescripción obligatoria con DCI en el ámbito institucional está contenida en las regulaciones de la mayoría de los países estudiados, excepto en Venezuela y Barbados. Además, excepto en Barbados, todas las regulaciones analizadas contemplan la obligatoriedad de usar la DCI en la compra de medicamentos por parte de los hospitales del sector público. Este parámetro no estuvo disponible en México y Nicaragua. Mientras, solo en cinco de los países estudiados (Argentina, Ecuador, Panamá, Paraguay y Perú) es obligatorio utilizar la DCI para prescribir medicamentos en las instituciones privadas.

No obstante, en el marco de la adopción de acuerdos comerciales en algunos países de la Región se analiza actualmente la propuesta de establecer restricciones en el uso de la DCI, invocando el artículo 20 del ADPIC (4). Ese artículo establece límites a las exigencias que los gobiernos pueden imponer para el etiquetado de bienes -incluidos los medicamentos-con vistas a evitar complicaciones injustificadas en el adecuado uso de una marca sin afectar a la diferenciación del producto. Esta tendencia podría entorpecer la aplicación de las recomendaciones sanitarias internacionales relacionadas con este tema (1-3).

\section{Definición de medicamento genérico}

La simple revisión de los documentos regulatorios no permite establecer de forma cabal la razón y el sentido de la adopción de una $\mathrm{u}$ otra definición de medicamento genérico. Sin embargo, la diversidad de definiciones adoptadas por los países (cuadro 2) podría estar relacionada con el desarrollo de los mercados farmacéuticos nacionales y sus políticas de estímulo a la competencia. En este sentido, la definición de medicamento genérico, más que un fin podría ser el medio utilizado por los países para consolidar la oferta de competidores.

Los resultados de este estudio indican que la promoción del uso de la DCI, la diversidad en los enfoques sobre la exigencia de estudios de BE y las restricciones o los incentivos a la sustitución de los productos prescritos por alternativas de menor costo son elementos que determinan la adopción y la complejidad de una $u$ otra definición de medicamento genérico y la aparición de otros términos, en algunas ocasiones mediante sinónimos como "medicamentos similares" o "copias", por una parte, y "productos originales", "innovadores" o "de marca", por otra.

Se debe reiterar que, aunque se reconoce que hay discrepancias en torno a la definición $(10,11)$, la concepción clásica establece que medicamento genérico es el que se comercializa después de la caducidad de la patente que lo cubre (6). No obstante, esta definición no se ha aplicado consecuentemente en América Latina y el Caribe debido a la expedición relativamente reciente de las normas sobre el reconocimiento de patentes en estos países (24).

En cuatro países (Bolivia, Costa Rica, Ecuador y Perú) se adoptó una definición simple de medicamento genérico, que establece que es el que se comercializa con su DCI. En Panamá, la Ley 1 de 2001 establecía que medicamento genérico es el que se distribuye o expende rotulado con el nombre genérico del principio activo. Sin embargo, una reglamentación aprobada en 2005 acercó la definición de medicamento genérico a la establecida en las regulaciones de Brasil y México (cuadro 2).

Tal vez una definición sencilla puede reflejar más fielmente la realidad y la intención de las políticas de competencia en los países estudiados, ya que el surgimiento de los medicamentos competidores no estuvo asociado con el vencimiento de las patentes ni con el cumplimiento de las pruebas específicas de BE terapéutica. 
CUADRO 2. Definición de medicamento genérico y términos asociados en los documentos regulatorios de los países estudiados. Abril, 2005

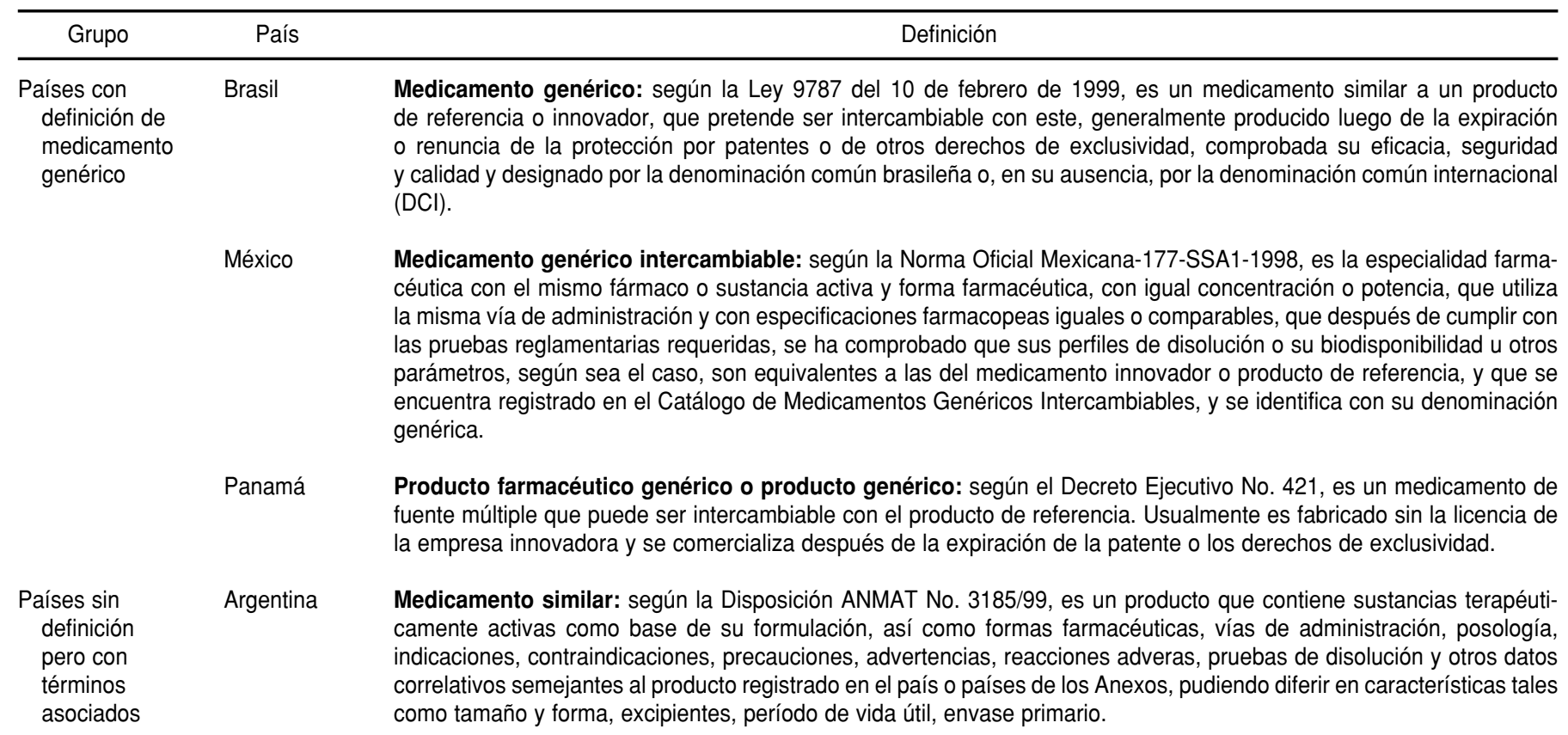

Costa Rica Medicamento o producto farmacéutico multiorigen: según Art. 3 del Decreto No. 28466-S, son medicamentos farmacéuticamente equivalentes que pueden o no ser equivalentes terapéuticamente. Cuando son equivalentes terapéuticos son intercambiables.

Medicamento de nombre genérico: medicamento que se distribuye o expende rotulado con el nombre común del principio activo, o sea, sin identificarse con una marca de fábrica o nombre comercial.

Colombia Medicamento competidor: según la Resolución 1400 de 2001, es el producto farmacéutico que contiene un principio activo que ya ha sido aceptado en las Normas Farmacológicas Colombianas y no es aquel producto con el cual se ha desarrollado la investigación completa de su desarrollo, desde su síntesis química hasta su utilización clínica.

Países con definición simple de genérico

Medicamento genérico: según el Decreto Supremo D.S. 010-97-SA, es el producto farmacéutico cuyo nombre corresponde a la $\mathrm{DCl}$ del principio activo, recomendada por la Organización Mundial de la Salud, y no es identificado con un nombre de marca.

Bolivia

A los fines reglamentarios, los medicamentos reconocidos por la Ley No. 1737 de 17 de diciembre de 1996 son: a) medicamentos genéricos (con $\mathrm{DCl}$ ) y b) medicamentos de marca comercial, entre otros.

Ecuador Medicamento genérico: según la Ley 2000-12, debe entenderse como medicamentos genéricos los que se registran y emplean con la DCl del principio activo, propuesta por la Organización Mundial de la Salud, o en su ausencia con una denominación genérica convencional reconocida internacionalmente, cuya patente de invención haya expirado. Estos medicamentos tendrán los mismos niveles de calidad, seguridad y eficacia requeridos para los de marca.

\begin{tabular}{|c|c|}
\hline \multirow{3}{*}{ Sin definiciones } & Barbados \\
\hline & Paraguay \\
\hline & Guatemala \\
\hline Sin información & Nicaragua \\
\hline & \\
\hline
\end{tabular}

Definiciones de la Organización Mundial de la Salud
Productos genéricos: el término producto genérico tiene diferentes significados en distintas jurisdicciones. El uso de este término se debe evitar tanto como sea posible, en su lugar se debe utilizar el término producto de fuentes múltiples. Los productos genéricos pueden comercializarse bajo la DCl o bajo un nombre de marca, en formas de dosificación o potencia diferente a la del innovador. Cuando el término producto genérico se usa, este significa un producto farmacéutico que usualmente se intenta intercambiar con el producto innovador, el cual se produce sin licencia de la compañía innovadora y se comercializa después de expirada la patente u otros derechos de exclusividad.

Productos de fuentes múltiples: son productos equivalentes farmacéuticos o alternativas farmacéuticas que pueden o no ser equivalentes terapéuticos. Los productos farmacéuticos de fuentes múltiples que son equivalentes terapéuticos son intercambiables. 
CUADRO 3. Criterios utilizados en las definiciones de medicamentos competidores

\begin{tabular}{lcc}
\hline \multicolumn{1}{c}{ Criterios comunes } & Criterios variables & Categorías \\
\hline Igual principio activo & Forma de comercialización & Con la denominación común internacional \\
Igual concentración o potencia & & Otra denominación común aprobada \\
Igual vía de administración & Equivalencia terapéutica & Marca o nombre propio \\
Igual uso & & Exigencia de estudios de bioequivalencia \\
& & Aceptación de pruebas diferentes a los \\
& & estudios de bioequivalencia (exenciones) \\
& Exclusividad en la comercialización & Ningún requisito de equivalencia \\
& & Patentes o protección de datos \\
& & Ninguna
\end{tabular}
a Protección de datos: se refiere a la exclusividad otorgada a nuevos principios activos mediante la protección de los estudios de seguridad y eficacia presenta-
dos en el proceso de registro sanitario.

La diversidad en las definiciones dificulta la caracterización de los mercados y las comparaciones, tanto de los países como de los diversos mercados dentro de un mismo país. En un estudio realizado para evaluar el desarrollo del mercado de medicamentos competidores en los países miembros de la Asociación Latinoamericana de Integración (ALADI) se encontraron dificultades para estimar el mercado de competidores debido a la diversidad de definiciones y a la falta de datos desagregados que respondan a dichas definiciones ${ }^{4}$.

Esta heterogeneidad también puede contribuir a la segmentación del mercado farmacéutico. Recientemente la firma International Measurements Statistics (IMS), principal fuente privada de información sobre datos de mercado farmacéutico, declaró que en los mercados latinoamericanos se comercializa una gran cantidad de productos denominados genéricos de marca y productos copia (similares) que no son bioequivalentes y que se utilizan ampliamente en el sector público (25). En su declaración, el IMS argumenta que las políticas gubernamentales se han orientado en los últimos años a pro-

\footnotetext{
4 Sánchez D. Exploración de las posibilidades de cooperación OPS/ALADI, en el desarrollo del mercado de medicamentos genéricos en sus países miembros [documento borrador]. OPS; 2004.
}

mover el mercado de competidores sin marca, pero destaca que en México se exige al sector público comprar preferentemente los llamados medicamentos competidores intercambiables (24).

Con esto se pone de relieve un tipo de segmentación del mercado farmacéutico, entre muchos otros existentes, que puede inducir a percepciones distorsionadas: medicamentos de primera categoría (originales o innovadores), de segunda (competidores intercambiables) y de tercera (similares o copias). Esta distorsión puede provocar problemas en el acceso, debido a la diferenciación en los precios y en la información.

Lo expuesto hasta aquí refuerza la necesidad de armonizar las definiciones de medicamento competidor (26). Desde 1996, la OMS - reconociendo la polémica asociada con la definición de estos medicamentos- recomendó utilizar el término "producto de fuentes múltiples" para referirse a los medicamentos competidores. Adicionalmente, recomendó el término "producto genérico" para referirse a los productos farmacéuticos intercambiables con el producto innovador, que se fabrican sin licencia de la empresa innovadora tras haber vencido la patente $\mathrm{u}$ otros derechos de exclusividad (11). En 2006, la OMS amplió esa definición e indicó que los productos de fuentes múltiples son equivalentes farmacéu- ticos o alternativas farmacéuticas que pueden ser equivalentes terapéuticos o no, mientras que los productos farmacéuticos de fuentes múltiples que son equivalentes terapéuticos son intercambiables (23). En esa ocasión, la OMS llamó la atención sobre el hecho de que el término "producto genérico" tiene diferentes significados en diferentes jurisdicciones, por lo que ha evitado su uso a favor del término "fuentes múltiples".

Sería recomendable desarrollar instrumentos que permitan comparar, comprender y prever el efecto que tienen las diferentes propuestas de armonización de estas definiciones sobre las políticas de competencia en el sector farmacéutico, en particular en la evaluación de los mercados.

\section{Requisito de realizar pruebas de $\mathrm{BE}$}

Al igual que ocurre con la definición de medicamento genérico, en los diferentes países se observó una gran heterogeneidad con relación a los requisitos de realizar pruebas de equivalencia terapéutica, en particular estudios de BE.

En los países en los que se aceptan las patentes y otras formas de exclusividad desde hace muchos años, las pruebas de BE se consideran parte de los parámetros de calidad de los medicamentos 
competidores y se utilizan como criterio de su desempeño clínico, por lo que los médicos se han familiarizado únicamente con el producto innovador durante el tiempo de exclusividad (27).

La reglamentación de la exigencia de realizar pruebas de BE constituye uno de los elementos de las políticas relacionadas con los medicamentos competidores (9). En España, por ejemplo, desde 1990 se estableció que la especialidad farmacéutica genérica debe demostrar la equivalencia terapéutica con la especialidad de referencia mediante los correspondientes estudios de $\mathrm{BE}$ (28). Sin embargo, los países sin regulaciones sobre el reconocimiento de patentes consolidaron sus industrias de productos competidores al margen de esta exigencia y los médicos usaron de forma simultánea los diferentes productos comercializados.

En Colombia, por ejemplo, en 1963 se reglamentó la expedición de licencias, la fabricación y la venta de productos farmacéuticos básicos bajo su nombre genérico o técnico con el propósito de reducir el precio de esos medicamentos (29). Más tarde, en 1992, se reglamentó la producción y el expendio de los medicamentos esenciales del Formulario Nacional bajo su nombre genérico (30). La Ley 100 de 1993 estableció que el Sistema General de Seguridad Social de Salud de Colombia debe crear las condiciones de acceso a un plan obligatorio de salud para todas las personas en el territorio nacional antes del año 2001 y proveer los medicamentos esenciales en su presentación genérica (16).

El perfeccionamiento de la industria farmacéutica colombiana, en términos de calidad, se llevó a cabo mediante la certificación de las buenas prácticas de manufactura, cuyo cumplimiento es obligatorio para todas las plantas de producción del país. Las normas que establecen la realización de las pruebas de BE se pusieron en vigor solo a partir de 2001 (31). No obstante, según la Asociación de Industriales Farmacéuticos (ASINFAR) de Colombia, las exportaciones de medicamentos a países de la región andina y a los Estados Unidos se incrementó de forma abrupta de 27,1 millones de dólares estadounidenses en 1991 a 248,5 millones de dólares en 2002 (A. Bravo, comunicación personal, 2003).

En los países donde las regulaciones de patentes son de reciente expedición, las modalidades de prescripción de medicamentos son tan diversas como alternativas existan simultáneamente en el mercado. En esas condiciones, la posible incertidumbre sobre el desempeño clínico de los medicamentos competidores tendrá que resolverse en la práctica cotidiana, ya que en muchos casos para su registro sanitario se aceptan los estudios clínicos de los productos innovadores de acuerdo con los principios éticos de la investigación clínica, según los cuales no deben realizarse estudios sobre lo ya probado.

Las pruebas de BE se establecieron como parte determinante de las políticas de competencia en 7 de los 14 países analizados entre 1999 (Argentina) y 2005 (Costa Rica), lo que demuestra que las pruebas de BE no se consideraban un elemento clave de las políticas de promoción de la competencia como se consignaba en algunos documentos $(32,33)$. En la actualidad, los países reglamentan la exigencia de las pruebas de $\mathrm{BE}$ de formas diversas: algunos analizan las solicitudes caso a caso, otros siguen los criterios establecidos por una comisión asesora nacional y otros aplican guías nacionales (12).

En un aporte relevante y novedoso en la Región de las Américas, en Argentina, Colombia y Costa Rica se toma en cuenta el riesgo clínico y sanitario de los medicamentos para establecer la exigencia de estudios de $\mathrm{BE}$ (10) y se establecieron listas reducidas de los medicamentos que requieren dichos estudios. En contraste, en Brasil y México se exigen estos estudios a una lista mucho más amplia de medicamentos, enfoque similar al adoptado por los Estados Unidos de América y Canadá. De una lista de 96 medicamentos, en los Estados Unidos se solicitan pruebas de BE a 88, en Canadá a 60, en México a 39 y en Brasil a 32. En Costa Rica y Colombia se exigen esas pruebas a la menor cantidad de ingredientes activos ( 7 y 5 , respectivamente) (9).

\section{Promoción de la sustitución}

La estrategia de sustitución de medicamentos basada en la promoción de la dispensación del producto de menor precio para mejorar la cobertura de medicamentos ha pasado por diferentes etapas: desde la presión de las regulaciones y las políticas gubernamentales sobre el dispensador, hasta la sugerencia del médico y la obligación incondicional de hacerlo (6).

En los Estados Unidos, una vez cumplidos los requisitos del registro, los productos competidores bioequivalentes se incorporan a una lista pública (Libro Naranja), publicada por la Administración de Alimentos y Medicamentos (FDA), que autoriza la sustitución del medicamento innovador por el competidor en el momento de la dispensación (34). En Brasil y México, siguiendo ese modelo, se aprobaron listas nacionales de medicamentos autorizados para la sustitución. Los medicamentos autorizados se deben prescribir y comercializar con la DCI y no deben estar protegidos por patentes. En esos tres países, la definición de medicamento genérico cobra sentido en el momento de la dispensación, pues la posibilidad de sustitución queda restringida a los productos competidores que cumplen con todos los criterios de la definición: el vencimiento de la patente, su comercialización con la DCI y la demostración de su equivalencia terapéutica con el medicamento innovador (cuadro 2).

En el caso de México, la lista de competidores - conocida como Libro Azul - permite al dispensador, al médico y al usuario conocer cuáles productos pueden sustituirse. En Brasil, la lista oficial de productos competidores, disponible en todas las farmacias, garantiza la sustitución únicamente de dichos productos (20). Siguiendo el mismo principio, en Panamá (22) y Venezuela (35) se han establecido plazos para la elaboración y adopción de la lista de productos que pueden sustituirse.

Para facilitar la identificación de estos medicamentos, en Brasil, México y Panamá se han establecido normas específicas para la rotulación de estos 
productos (cuadro 2). En México, los productos que cumplen con los requisitos establecidos se denominan genéricos intercambiables y se deben rotular con su DCI y las letras "GI" en amarillo, mientras en Brasil, estos productos llevan en su rótulo la letra " $\mathrm{G}$ " resaltada en una banda amarilla y en Panamá, los medicamentos intercambiables se identifican mediante las letras "MI" en su rótulo.

\section{CONCLUSIONES}

En los países analizados se han realizado avances importantes en el uso de la DCI en los medicamentos competidores, particularmente en su rotulado y su prescripción en las instituciones públicas de salud. A pesar de que este es un elemento determinante de las políticas de competencia, los mecanismos de promoción de la DCI en el ámbito privado y los incentivos económicos son poco evidentes en las regulaciones consultadas. Sin embargo, se debe tener presente que los procesos de negociación de los tratados de libre comercio en la Región pueden detener o revertir los avances en esta materia.

Las directivas de la OMS y la OPS sobre la necesidad de realizar estudios de $B E$ constituyen una referencia importante, sin embargo, las regulaciones relacionadas con la definición de medicamento competidor, los estudios de BE y la sustitución de medicamentos innovadores con medicamentos competido- res muestran marcadas diferencias entre los países estudiados. Por ello, los esfuerzos de armonización deberán tomar en cuenta la posible relación entre las definiciones adoptadas por los países, el desarrollo de los mercados farmacéuticos nacionales y sus políticas para incentivar la competencia.

La principal limitación metodológica del presente estudio es la posible pérdida de vigencia de estos resultados por cambios en las regulaciones nacionales. A pesar de los esfuerzos realizados para hacer la búsqueda exhaustiva, es posible que no se hayan analizado todas las normas y documentos oficiales pertinentes. Además, la interpretación de las normas se realizó desde el punto de vista regulatorio y de armonización y según el conocimiento y la experiencia de los autores.

A pesar de que la calidad percibida es uno de los factores que determinan la aceptación de un medicamento y puede constituir un problema importante en algunos países de la Región de las Américas y que las recomendaciones internacionales establecen la necesidad de aplicar estrategias que mejoren la aceptación de los medicamentos competidores, esta variable no se evaluó en este estudio.

Los resultados expuestos indican que en los países estudiados se observan tres tendencias regulatorias: los países que favorecen la financiación de medicamentos competidores, la promoción extendida del uso de la DCI y no ponen restricciones a la sustitución de medicamentos innovadores por competidores (Argentina, Colombia, Costa Rica, Ecuador y Paraguay); los que cuentan con un esquema orientado a la demostración de la equivalencia terapéutica, que se traduce en la restricción de la sustitución a los productos autorizados en listas de medicamentos competidores autorizados, prescritos mediante su DCI y con rótulos distintivos (Brasil, México, Panamá y Venezuela); y los que se encuentran en una etapa incipiente de su proceso de regulación (Barbados, Bolivia, Guatemala, Nicaragua y Perú).

Tomando en cuenta estas tendencias, se recomienda realizar un análisis estructurado sobre el acceso, el comportamiento de los precios del mercado farmacéutico, las percepciones y la aceptabilidad de los medicamentos competidores. Con esa información se podrán hacer recomendaciones regulatorias más coherentes que permitan a los países desarrollar estrategias de competencia que mejoren el acceso de la población a los medicamentos.

Agradecimientos. Para la realización de este documento se contó con la participación de los profesionales técnicos de la OPS encargados del tema de medicamentos en los países estudiados. El diseño y análisis de la información se realizó en la Fundación IFARMA y en la sede de la OPS en Washington, Estados Unidos. Se agradece a todos los que colaboraron en la revisión y recolección de la información, en especial al químico farmacéutico Freddy Murcia.

\section{REFERENCIAS}

1. Organización Panamericana de la Salud. Acceso a los medicamentos. Resolución CD45/10 del Consejo Directivo de la OPS. Washington, D.C.: OPS; 2004. Hallado en: http://www.paho.org/Spanish/GOV/CD/ cd45-10-s.pdf. Acceso el 7 de agosto de 2005.

2. World Health Organization. How to develop and implement a national drug policy. 2nd edition. Geneva: WHO; 2001. Pp. 34-5.

3. World Health Organization. WHO medicine strategy: framework for action in essential drugs and medicines policy 2000-2003. Geneva: WHO; 2000.
4. Correa CM. Patentes, industria farmacéutica y biotecnología. Rev Derecho Industrial. 1990; 12(35):335-43.

5. Organización Mundial del Comercio. Acuerdo sobre los Aspectos de los Derechos de Propiedad Intelectual Relacionados con el Comercio. Ginebra: OMC; 1995. Hallado en: http://www. oepm.es/internet/legisla/dcho_ int/14adpic. htm. Acceso el 7 de agosto de 2006.

6. Lobo F, Velásquez G. Los medicamentos ante las nuevas realidades económicas. Madrid: Civitas; 1997. Pp. 246.
7. Organización Mundial de la Salud, Comité de Expertos en Especificaciones para las Preparaciones Farmacéuticas. Informe 32. Ginebra: OMS; 1992.

8. Organización Panamericana de la Salud. Elementos técnicos de una política de medicamentos genéricos. Washington, D.C.: OPS; 1993.

9. Red Panamericana para la Armonización de la Reglamentación Farmacéutica, Grupo de Bioequivalencia. Criterios científicos para los ensayos de bioequivalencia "in vivo" e "in vitro", las bioexenciones y las estrategias para su implementación [documento en discusión]. 
Washington, D.C.: OPS; 2005. Hallado en http://www.paho.org/spanish/ad/ths/ev/ bedocumentocientificoborradorespanol.pdf. Acceso el 7 de agosto de 2006.

10. Homedes N, Ugalde A. Multisource drug policies in Latin America: survey of 10 countries. Bull World Health Organ. 2005; 83:64-70.

11. World Health Organization Expert Committee on Specifications for Pharmaceutical Preparations. Thirty-fourth report. Geneva: WHO; 1996. (WHO Technical Report Series No. 863).

12. World Health Organization. Marketing authorization of pharmaceutical products with special reference to multisource (generic) products. Geneva: WHO; 1999. (Regulatory Support Series No. 5).

13. República Argentina, Poder Ejecutivo. Decreto No. 486 (PEN) del 13 de marzo de 2002. Declárase la emergencia sanitaria nacional. Boletín Oficial de la República Argentina 2002 13 de marzo.

14. República Argentina, Ministerio de Salud. Resolución 201 del 19 de abril de 2002. Apruébase el Programa Médico Obligatorio de Emergencia (PMOE) integrado por el conjunto de prestaciones básicas esenciales garantizadas por los agentes del seguro de salud comprendidos en el artículo $1 .^{\circ}$ de la Ley $\mathrm{N}^{\circ}$ 23.660. Boletín Oficial de la República Argentina 200219 de abril.

15. República Argentina, Superintendencia de Servicios de Salud. Resolución 163 del 13 de mayo de 2002. Establécese que los descuentos a favor de los beneficiarios de los medicamentos en la modalidad ambulatorio solo serán reconocidos y aplicados por los agentes respecto de aquellas recetas que emitan por nombre genérico del principio activo. Boletín Oficial de la República Argentina 200213 de mayo.

16. República de Colombia. Ley 100 de 1993 del 23 de diciembre de 1993. Por la cual se crea el sistema de seguridad social integral y se dictan otras disposiciones. Diario Oficial de la República de Colombia; 1993. (D.C. No. 41.148). Hallado en: http://www.secretaria senado.gov.co/leyes/L0100_93.htm. Acceso el 5 de octubre de 2006

17. Organização Pan-Americana da Saúde. Assistência farmacêutica para gerentes municipais. Brasília, D.F.: OPAS; 2003.

18. República de Costa Rica, Asamblea Legislativa. Ley No. 17 del 22 de octubre de 1943. Ley constitutiva de la Caja Costarricense de Seguro Social. San José: Asamblea Legislativa; 1943. Hallado en: http://www.asamblea.go. cr/ley/leyes/1000/17.doc. Acceso el 5 de octubre de 2006.

19. República de Ecuador. Ley 2000-12 del 17 de abril de 2000. Ley de producción, importa- ción, comercialización y expendio de medicamentos genéricos de uso humano. Registro Oficial de la República de Ecuador 200017 de abril.

20. República Federativa de Brasil, Agencia Nacional de Vigilancia Sanitaria. Resolução RDC No. 135 de 29 de maio de 2003e. Aprova regulamento técnico para medicamentos genéricos. Diário Oficial [da] República Federativa do Brasil 200302 junio. Hallado en: http:// e-legis.bvs.br/leisref/public/showAct.php? id=7909. Acceso el 5 de octubre de 2006.

21. Estados Unidos Mexicanos. Norma Oficial Mexicana NOM-177-SSA1-1998 del 12 de abril de 1999. Que establece las pruebas y procedimientos para demostrar que un medicamento es intercambiable. Requisitos a que deben sujetarse los terceros autorizados que realicen las pruebas. Diario Oficial de la Federación 199907 mayo. Hallado en: http://www. salud.gob.mx/unidades/cdi/nom/177ssa18. $\mathrm{html}$. Acceso el 16 de octubre de 2006.

22. República de Panamá. Decreto Ejecutivo No. 421 del 24 de noviembre de 2004. Por el cual se deroga el Decreto Ejecutivo 331 de 30 de agosto de 2004, que modificó el Decreto Ejecutivo 65 de 6 de mayo de 2002 y complementó las disposiciones del Capítulo IV de la Ley 1 de 10 de enero de 2001, relativo a la equivalencia y eficacia terapéutica y se adoptan otras disposiciones. Gaceta Oficial de la República de Panamá 200430 de noviembre.

23. World Health Organization Expert Committee on Specifications for Pharmaceutical Preparations. Fortieth report. Geneva: WHO; 2006. (WHO Technical Report Series No. 937).

24. Oliveira MA, Bermúdez J, Velásquez G. Has the implementation of the TRIPS Agreement in Latin America and the Caribbean produced intellectual property legislation that favours public health? Bull World Health Organ. 2004;82(11):1-7.

25. International Measurements Statistics. Latin America: overcoming economic challenges. Fairfield, Connecticut, USA: IMS; 2006. Hallado en: http://www.imshealth.com/web/ content/0,3148,64576068_63872702_70260998 _71226846,00.html. Acceso el 5 de octubre de 2006.

26. Organización Panamericana de la Salud. Informe del Grupo de Trabajo sobre los Aspectos de los Derechos de Propiedad Intelectual Relacionados con el Comercio (ADPIC) y el Acceso a Medicamentos. Washington, D.C.: OPS; 2006.

27. Mossinghoff GJ. Overview of the HatchWaxman Act (1984) and its impact on the drug development process. Food Drug Law J. 2005;54:187-94

28. España. Ley 25 del 20 de diciembre de 1990. Del medicamento. Boletín Oficial del
Estado 199022 de diciembre. (RCL 1990, 2643). Hallado en: http://sia.juntaex.es/ pdfs/legislacion/LeyEst25-90.pdf. Acceso el 5 de octubre de 2006.

29. República de Colombia, Ministerio de Salud. Decreto 0291 de 1963. Por el cual se reglamenta la expedición de licencias, la fabricación y la venta de productos farmacéuticos básicos bajo su nombre genérico o técnico, con el propósito de realizar el plan de abaratamiento en el precio de las drogas y se dictan otras disposiciones. Santa Fe de Bogotá: Ministerio de Salud; 1963.

30. República de Colombia, Ministerio de Salud. Decreto 709 de 1992. Por el cual se reglamenta la producción y expendio de los medicamentos esenciales del Formulario Nacional bajo su nombre genérico. Santa Fe de Bogotá: Ministerio de Salud; 1992.

31. República de Colombia, Ministerio de Salud. Resolución 1400 de 24 de agosto de 2001. Por la cual se establece la Guía de Biodisponibilidad y de Bioequivalencia de Medicamentos de que trata el Decreto 677 de 1995. Santa Fe de Bogotá: Ministerio de Salud de Colombia; 2001.

32. República Argentina, Ministerio de Salud. Disposición ANMAT No. 3185/99 del 25 de junio de 1999. Apruébanse las recomendaciones técnicas contenidas en el documento Cronograma para exigencias de estudios de equivalencia entre medicamentos de riesgo sanitario significativo. Boletín Oficial de la República Argentina 199902 de julio. Hallado en: http://www.anmat.gov.ar/normativa/ Normativa/Medicamentos/Disposicion_ ANMAT_3185-1999.pdf. Acceso el 5 de octubre de 2006.

33. República de Costa Rica, Ministerio de Salud. Decreto 32470 S del 10 de junio de 2005. Reglamento para el registro sanitario de los medicamentos que requieren demostrar equivalencia terapéutica. La Gaceta 20054 de agosto. Hallado en: http:/ / www.hacienda.go.cr/centro/ datos / Decreto/Decretos-La \%20Gaceta \% 20149-4\%20AGO-2005.doc. Acceso el 5 de octubre de 2006.

34. U.S. Food and Drug Administration. Orange Book. Rockville, Maryland, USA: FDA; 2006. Hallado en http://www.fda.gov/cder/ob/ docs/preface/eclink.htm. Acceso el 5 de octubre de 2006.

35. República de Venezuela. Ley de Medicamentos. Gaceta Oficial 20003 de agosto.

Manuscrito recibido el 9 de febrero de 2006. Aceptado para publicación, tras revisión, el 26 de julio de 2006. 
ABSTRACT Objectives. To characterize current regulatory practices and trends relating to competing medications in 14 Latin American and Caribbean countries.

Defining generic drugs:

Methods. Between July 2004 and April 2005 we collected information on national regulations and policies that established or contained a definition of "generic drug" and related terms in 14 Latin American and Caribbean countries: Argentina, Barbaan end or a means? Analysis of regulations in 14 countries in the Region of the Americas dos, Bolivia, Brazil, Colombia, Costa Rica, Ecuador, Guatemala, Mexico, Nicaragua, Panama, Paraguay, Peru, and Venezuela. We also compiled information on the incentives to register and produce competing medications, promotion of the use of International Nonproprietary Names (INNs) for pharmaceutical substances, the regulation of bioequivalence studies, and the replacement of prescription medications with less expensive alternatives.

Results. We noted three patterns in the regulations: (1) countries that encourage financial support for competing medications, extensively promote the use of INNs, and place no restrictions on the replacement of innovative (proprietary) medications with competing products (Argentina, Colombia, Costa Rica, Ecuador, and Paraguay); (2) countries with policies aimed at showing therapeutic equivalence, which limits substitutions to approved products from a list of authorized competing medications that must be prescribed by their INN and must use distinctive labeling (Brazil, Mexico, Panama, and Venezuela); and (3) countries at a beginning stage in the process of regulation (Barbados, Bolivia, Guatemala, Nicaragua, and Peru). This variety leads to difficulties in characterizing pharmaceutical markets, and can lead to misleading categorizations.

Conclusions. Efforts to harmonize policies should consider the possible relationship among definitions adopted in different countries, the development of national pharmaceutical markets, and national policies to stimulate competition.

Key words Drug industry; drugs, generic; drug and narcotic control; Americas. 